\title{
Stability of magnetic configurations containing the toroidal and axial fields
}

\author{
A. Bonanno ${ }^{1,2}$ and V. Urpin ${ }^{1,3}$ \\ 1 INAF - Osservatorio Astrofisico di Catania, via S. Sofia 78, 95123 Catania, Italy \\ e-mail: abo@oact.inaf.it \\ 2 INFN, Sezione di Catania, via S. Sofia 72, 95123 Catania, Italy \\ 3 A.F. Ioffe Institute of Physics and Technology and Isaac Newton Institute of Chile, Branch in St. Petersburg, 194021 St. Petersburg, \\ Russia
}

Received 14 March 2007 / Accepted 18 September 2007

\begin{abstract}
Stability properties of magnetic-field configurations containing the toroidal and axial field are considered. The stability is treated by making use of linear analysis. It is shown that the conditions required for the onset of instability are essentially different from those given by the necessary condition $\mathrm{d}\left(s B_{\varphi}\right) / \mathrm{d} s>0$, where $s$ is the cylindrical radius. The growth rate of instability is calculated for a wide range of the parameters. We argue that the instability can operate in two different regimes depending on the strength of the axial field and the profile of the toroidal field.
\end{abstract}

Key words. magnetohydrodynamics (MHD) - instabilities - stars: magnetic fields

\section{Introduction}

Turbulence generated by MHD instabilities can play an important role in enhancing transport processes in various astrophysical bodies, such as accretion and protoplanetary disks, galaxies, stellar radiative zones, etc. The anomalous turbulent transport can be particularly important in magnetized gas where a wide variety of MHD instabilities can occur (see, e.g., Barnes et al. 1999). In this case, the onset of instability can be caused both by hydrodynamic motions (for instance, differential rotation; see, e.g., Velikhov 1959; Chandrasekhar 1960) or unstable magnetic configurations. Which field strength and topology can sustain a stable magnetic configuration is still rather uncertain despite extensive work (see Borra et al. 1982; Mestel 1999, for review).

Most likely, the best-studied magnetic configuration is one with a purely toroidal field. Ever since the paper by Tayler (1973), it has been known that toroidal fields can be unstable close to the axis of symmetry, if there is a non-zero electric current density on the axis. The growth rate of this instability is expected to be of the order of the time taken for an Alfvén wave to travel around the star on a toroidal field line. However, even a purely toroidal field is stable if it decreases rapidly with the cylindrical radius $s$. For instance, Tayler (1973; see also Chanmugam 1979) argued that the toroidal field $B_{\varphi}$ is stable against axisymmetric perturbations if it satisfies the condition $\mathrm{d}\left(B_{\varphi} / s\right) / \mathrm{d} s<0$ and to non-axisymmetric perturbations if $\mathrm{d}\left(s B_{\varphi}^{2}\right) / \mathrm{d} s<0$. Note that a purely toroidal field can also be subject to the magnetic buoyancy instability (Parker 1955; Gilman 1970; Acheson 1978) but the Tayler instability likely appears first as the strength of the toroidal field increases (Spruit 1999).

The stability of a purely toroidal field in the radiative zones of stars and accretion disks has been studied by a number of authors. Numerical modeling by Braithwaite (2006) confirms that the toroidal field with $B_{\varphi} \propto s$ or $\propto s^{2}$ is unstable to the $m=1$ mode ( $m$ is the azimuthal wave number) as predicted by
Tayler (1973). The linear stability of the toroidal field in rotating stellar interiors has been considered by Kitchatinov \& Rüdiger (2007), who conclude that the magnetic instability is essentially three-dimensional and that the finite thermal conductivity has a strong destabilizing effect. Terquem \& Papaloizou (1996) and Papaloizou \& Terquem (1997) considered the stability of an accretion disk with an embedded toroidal magnetic field. These authors find that the disks containing a purely toroidal field are always unstable and obtained spectra of unstable modes in the local approximation. They argue that one type of modes is driven primarily by buoyancy, while the other is driven by shear independently of the magnetic configuration.

Stability properties of purely poloidal magnetic configurations have also been well-studied. Since the papers by Wright (1973) and Markey \& Tayler $(1973,1974)$, it is known that a poloidal field is subject to dynamical instabilities in the neighbourhood of neutral points/lines if the field lines are closed inside the star. These authors recognizes that the magnetic field in the neighbourhood of a neutral line resembles that of a toroidal pinched discharge that is known to be unstable. Although instabilities involving significant displacements in the direction of gravity were strongy inhibited, other instabilities were not affected. The instability of poloidal configurations is rather fast: its growth time can reach a few Alfvén crossing time (Van Assche et al. 1982; Braithwaite \& Spruit 2006). With numerical simulations, the stability of poloidal magnetic configurations has been studied by Braithwaite \& Spruit (2006), who apply the results to the internal magnetic configuration of neutron stars. Note, however, that a toroidal field might exert a stabilising influence on the instabilities of a poloidal field in the neighbourhood of neutral points (Tayler 1980).

On the contrary, the addition of even a relatively weak poloidal field alters the stability of the toroidal field substantially. For example, as first shown by Howard \& Gupta (1962; see also Knobloch 1992; Dubrulle \& Knobloch 1993), 
a necessary (but not sufficient) condition for the instability of a toroidal field in the presence of the axial field reads

$$
\frac{\mathrm{d}}{\mathrm{d} s}\left(s B_{\varphi}\right)>0 \text {. }
$$

Howard \& Gupta (1962) argue that, for a fixed value of $m$, the growth rate of instability caused by condition (1) must vanish in the limit of a vanishing axial magnetic field, thereby providing a connection with the stability criterion obtained by Tayler (1973). Note that the presence of a radial field is also crucial for stability properties of rotating magnetic configurations (Bonanno \& Urpin 2006). It turns out that configurations containing both toroidal and poloidal fields are more stable than purely toroidal or purely poloidal ones (Prendergast 1956; Tayler 1980). With numerical simulations, Braithwaite \& Nordlund (2006) studied the stability of a random initial field in the stellar radiative zone. The star was modeled on a Cartesian grid, and the authors found that the stable magnetic configurations generally have the form of tori with comparable poloidal and toroidal field strengths.

In the present paper, we address the stability properties of magnetic configurations by considering the stability of the toroidal magnetic field with respect to axisymmetric perturbations in the presence of axial fields of a various strength. We show that the instability may occur in such magnetic field configurations under the conditions that differ substantially from those imposed by the Tayler criterion or the necessary condition (1). We argue that, in some cases, the instability is caused by the new type of MHD waves with the growth rate proportional to $\sqrt{B_{z} B_{\varphi}}$ where $B_{z}$ is the axial magnetic field. Depending on the profile $B_{\varphi}(s)$ and the ratio $B_{z} / B_{\varphi}$, the instability can occur in two regimes that have substantially different growth rates. We also show that the range of unstable wavelength in the $z$-direction can be essentially different, depending on the $B_{z} / B_{\varphi}$ ratio.

\section{Basic equations}

Let us consider the stability of an axisymmetric cylindrical magnetic configuration assuming plasma to be conducting perfectly. We work in cylindrical coordinates $(s, \varphi, z)$ with the unit vectors $\left(\boldsymbol{e}_{s}, \boldsymbol{e}_{\varphi}, \boldsymbol{e}_{z}\right)$. The inner and outer radii of the configuration are $s_{1}$ and $s_{2}$, respectively ( $s_{1}$ can be equal to 0 ). We assume that the axial magnetic field $B_{z}$ and the azimuthal field $B_{\varphi}$ depend on the cylindrical radius alone: $B_{z}=B_{z}(s)$ and $B_{\varphi}=B_{\varphi}(s)$. Usually, the axial field in our model can even change the sign at some point $s_{0}$ within $\left(s_{1}, s_{2}\right), s_{2}>s_{0}>s_{1}$. Such a dependence is considered in order to mimic the stability properties of stars where the axial field component can generally change the sign.

The equations of incompressible MHD are

$$
\frac{\partial \boldsymbol{v}}{\partial t}+(\boldsymbol{v} \cdot \nabla) \boldsymbol{v}=-\frac{\nabla p}{\rho}+\frac{1}{4 \pi \rho}(\nabla \times \boldsymbol{B}) \times \boldsymbol{B},
$$

$\nabla \cdot v=0$

$$
\frac{\partial \boldsymbol{B}}{\partial t}-\nabla \times(\boldsymbol{v} \times \boldsymbol{B})=0,
$$

$\nabla \cdot \boldsymbol{B}=0$.

It is assumed that gas is in hydrostatic equilibrium in the basic state and that there is no rotation, so

$$
\frac{\nabla p}{\rho}=\frac{1}{4 \pi \rho}(\nabla \times \boldsymbol{B}) \times \boldsymbol{B} .
$$

In this paper we consider the stability of axisymmetric perturbations. Since the basic state is stationary, the time dependence of perturbations can be taken in the form exp $\sigma t$. Small perturbations will be indicated by subscript 1 , while unperturbed quantities will have no subscript. The linearized MHD equations are

$$
\begin{aligned}
& \sigma \boldsymbol{v}_{1}=-\frac{\nabla p_{1}}{\rho}+\frac{1}{4 \pi \rho}\left[\left(\nabla \times \boldsymbol{B}_{1}\right) \times \boldsymbol{B}+(\nabla \times \boldsymbol{B}) \times \boldsymbol{B}_{1}\right], \\
& \nabla \cdot \boldsymbol{v}_{1}=0, \\
& \sigma \boldsymbol{B}_{1}-(\boldsymbol{B} \cdot \nabla) \boldsymbol{v}_{1}+\left(\boldsymbol{v}_{1} \cdot \nabla\right) \boldsymbol{B}=0, \\
& \nabla \cdot \boldsymbol{B}_{1}=0 .
\end{aligned}
$$

Equations (7)-(10) are homogeneous in $z$ and admit solutions in the form of waves in the $z$-direction, $\propto \exp (-\mathrm{i} k z)$ where $k$ is the wavevector. In stellar conditions, this analysis applies if $k$ satisfies the condition $k H>1$ where $H$ is the lengthscale of the basic state in the $z$-direction. Eliminating all variables in favour of the radial velocity perturbation $v_{1 s}$, we obtain the following differential equation

$$
\begin{array}{r}
\left(\sigma^{2}+\omega_{A}^{2}\right)\left[\frac{\mathrm{d}}{\mathrm{d} s}\left(\frac{\mathrm{d} v_{1 s}}{\mathrm{~d} s}+\frac{v_{1 s}}{s}\right)-k^{2} v_{1 s}\right]+\frac{4 k^{2} \omega_{A}^{2} \omega_{B}^{2}}{\left(\sigma^{2}+\omega_{A}^{2}\right)} v_{1 s} \\
-2 k^{2} \omega_{B}^{2}(1-\alpha) v_{1 s}=-\frac{2}{s} \delta \omega_{A}^{2}\left(\frac{\partial v_{1 s}}{\partial s}+\frac{v_{1 s}}{s}\right),
\end{array}
$$

where

$\omega_{A}=\frac{k B_{z}}{\sqrt{4 \pi \rho}}, \quad \omega_{B}=\frac{B_{\varphi}}{s \sqrt{4 \pi \rho}}, \quad \alpha=\frac{\partial \ln B_{\varphi}}{\partial \ln s}, \quad \delta=\frac{\partial \ln B_{z}}{\partial \ln s}$.

In the case $\delta=0$ ( $B_{z}=$ const.), Eq. (11) recovers the equation derived by Acheson (1973) and Knobloch (1992). Once the radial velocity is known, one can calculate the perturbations of other quantities. For example, a perturbation of the vertical field can be expressed in terms of $v_{1 s}$ as

$B_{1 z}=-\left(B_{z} / \sigma s\right) \partial\left(s v_{1 s}\right) / \partial s-v_{1 s} \frac{\partial B_{z}}{\partial s}$.

With appropriate boundary conditions, Eq. (11) allows the eigenvalues $\sigma$ to be determined. In this study, we choose the simplest boundary conditions and assume that the radial velocity vanishes at $s_{1}$ and $s_{2}$.

\section{Analytical consideration of Eq. (11)}

In this section, we consider few particular cases of Eq. (11) when it allows an analytical solution. The analytical consideration of simple cases can be a useful guide in understanding the stability properties of more complex field configurations as well as in the interpretation of numerical results.

\subsection{Instability in the case $B_{\varphi} \propto s$}

Let us initially consider the case of the azimuthal field being proportional to the cylindrical radius, $B_{\varphi}=B_{\varphi 0}\left(s / s_{1}\right)$, where $B_{\varphi 0}$ is the field strength at $s=s_{1}$. Then, the quantities $B_{\varphi} / s$ and $\omega_{B}$ are constant, and the last term on the left hand side of Eq. (11) vanishes. For the sake of simplicity, we also assume that the axial field is constant. After some algebra, Eq. (11) can be transformed into

$\frac{\mathrm{d}^{2} v_{1 s}}{\mathrm{~d} \xi^{2}}+\frac{1}{\xi} \frac{\mathrm{d} v_{1 s}}{\mathrm{~d} \xi}+\left(1-\frac{1}{\xi^{2}}\right)=0$ 
where

$\xi=k s \sqrt{F}, \quad F=\frac{4 \omega_{A} \omega_{B}^{2}}{\left(\sigma^{2}+\omega_{A}^{2}\right)^{2}}-1$.

The solution of this equation can be represented in terms of the Bessel functions of the order of 1 (see, e.g., Morse \& Feshbach 1953),

$v_{1 s}=C_{1} J_{1}(\xi)+C_{2} Y_{1}(\xi)$,

with $C_{1}$ and $C_{2}$ being constant. For the chosen boundary conditions, the eigenvalues of Eq. (13) are determined by the equation

$J_{1}\left(\xi_{1}\right) Y_{1}\left(p \xi_{1}\right)-Y_{1}\left(\xi_{1}\right) J_{1}\left(p \xi_{1}\right)=0$,

where $p=s_{2} / s_{1}$ and $\xi_{1}=k s_{1} \sqrt{F}$. The roots of Eq. (16) are real and simple. If $p>1$, the asymptotic expansion of the $n$th zero is

$\xi_{1}^{(n)} \approx \frac{n \pi s_{1}}{\Delta s}+\frac{3 \Delta s}{8 n \pi s_{2}}+\ldots, \quad \Delta s=s_{2}-s_{1}$

(see, e.g., Olver 1970). Since $\xi_{1}^{(n)}=k s_{1} \sqrt{F}$, we obtain the dispersion relation for $\sigma$ in the form

$\left(\sigma^{2}+\omega_{A}^{2}\right)^{2}=4 \mu \omega_{A}^{2} \omega_{B}^{2}$,

where $\mu=k^{2} /\left(k^{2}+k_{s}^{2}\right)$ and $k_{s}=\xi_{1}^{(n)} / s_{1}$. Then,

$\sigma^{2}=-\omega_{A}^{2} \pm 2 \sqrt{\mu} \omega_{A} \omega_{B}$.

One of the roots is positive if the toroidal field satisfies the inequality

$B_{\varphi}\left(s_{1}\right)>B_{z}\left(\frac{k s_{1}}{2 \sqrt{\mu}}\right)$

where $B_{\varphi}\left(s_{1}\right)$ is the strength of the toroidal field at the inner boundary. If condition (20) is fulfilled, then two aperiodic modes exist with

$\sigma_{1,2} \approx \pm \mu^{1 / 4} \sqrt{2 \omega_{A} \omega_{B}} \propto \sqrt{B_{z} B_{\varphi}}$

One of these modes is always unstable, and another one is stable and decays exactly on the timescale on which the unstable mode grows. The other two modes that exist in the considered magnetic configuration are also stable but oscillatory. Under condition (22), their frequencies are given approximately by

$\sigma_{3,4} \approx \pm i \mu^{1 / 4} \sqrt{2 \omega_{A} \omega_{B}}$

and are also $\propto \sqrt{B_{z} B_{\varphi}}$. To the best of our knowledge, the type of MHD wave with dispersion Eqs. (21) and (22) has not been considered in the literature yet. These modes exist in fluid only if both the axial and toroidal magnetic field are non-vanishing since their frequency (or growth/decay timescale) is proportional to $\sqrt{B_{z} B_{\varphi}}$. Note that if the toroidal field is weak and condition (20) is not satisfied, the modes given by Eq. (18) transform into ordinary Alfvén waves.

\subsection{Instability of short wavelength perturbations}

If the wavelength of perturbations is shorter than the characteristic lengthscale of unperturbed quantities, then one can neglect the terms of the order $1 / s$ compared to $\mathrm{d} / \mathrm{d} s$ in Eq. (11). In this case, Eq. (11) can be transformed into

$$
\begin{array}{r}
\frac{\mathrm{d}^{2} v_{1 s}}{\mathrm{~d} s^{2}}+\frac{2 \delta \omega_{A}^{2}}{s\left(\sigma^{2}+\omega_{A}^{2}\right)} \frac{\mathrm{d} v_{1 s}}{\mathrm{~d} s}-k^{2}\left[1-\frac{4 \omega_{A}^{2} \omega_{B}^{2}}{\left(\sigma^{2}+\omega_{A}^{2}\right)^{2}}\right. \\
\left.+\frac{2 \omega_{B}^{2}(1-\alpha)}{\left(\sigma^{2}+\omega_{A}^{2}\right)}\right] v_{1 s}=0 .
\end{array}
$$

In a short-wavelength approximation, the coefficients of this equation can be treated as constant, and the solution can be taken in the form $F \propto \exp \left(-\mathrm{i} k_{s} s\right)$ where $k_{s}$ is the wavevector in the radial direction. The dispersion relation corresponding to Eq. (23) is

$\left(\sigma^{2}+\omega_{A}^{2}\right)\left(\sigma^{2}+\omega_{A}^{2}+2 A\right)-4 \mu \omega_{A}^{2} \omega_{B}^{2}=0$,

where

$A=(1-\alpha) \mu \omega_{B}^{2}+\frac{i k_{s}}{s q^{2}} \delta \omega_{A}^{2}, \quad \mu=\frac{k^{2}}{Q^{2}}, \quad Q^{2}=k^{2}+k_{s}^{2}$.

The solution of Eq. (24) is

$\sigma^{2}=-\omega_{A}^{2}-A \pm \sqrt{A^{2}+4 \mu \omega_{A}^{2} \omega_{B}^{2}}$

If the azimuthal field is vanishing $\left(\omega_{B}=0\right)$, then the roots are

$\sigma_{1,2}^{2}=-\omega_{A}^{2}, \quad \sigma_{3,4}^{2}=-\omega_{A}^{2}-\frac{2 \mathrm{i} k_{s}}{s Q^{2}} \delta \omega_{A}^{2}$.

The first two modes are always stable. The modes 3 and 4 are usually stable since the second term in the expression for $\sigma_{3,4}$ is of the order of $1 /\left(s k_{s}\right) \ll 1$ compared to the first term and should be neglected in a short-wavelength approximation. The only exception is the region in a neighbourhood of the neutral point (or line) where $B_{z}(s) \rightarrow 0$ and, hence, $\omega_{A} \rightarrow 0$. For example, if $B_{z}(s)$ goes to zero $\propto\left(s-s_{0}\right)$, then the first term in $\sigma_{3,4}^{2}$ is $\propto\left(s-s_{0}\right)^{2}$, but the second one is $\propto\left(s-s_{0}\right)$. Therefore, the region always exists in the neighbourhood of $s_{0}$ where the second term dominates the first one. In this region, the roots 3 and 4 are approximately given by

$\sigma_{3,4} \approx \pm \frac{1-i}{Q} \sqrt{\frac{\delta k_{s}}{s}} \omega_{A}$

One of the roots (27) corresponds to the unstable mode. Therefore, poloidal magnetic configurations with the neutral point (line) are always unstable in the neighbourhood of this point (line). This fact was first pointed out by Tayler (1973).

However, the presence of the azimuthal field can drastically change the stability properties of the magnetic field even in a neighbourhood of the neutral point. Near the neutral point, the azimuthal field is stronger than the axial one (if the former does not have zero at the same point $s_{0}$ ), then from Eq. (25) we have with the accuracy in terms of the order of $\omega_{A}^{2}$

$\sigma_{1,2}^{2} \approx 2(\alpha-1) \mu \omega_{B}^{2}, \quad \sigma_{3,4}^{2} \approx \omega_{A}^{2} \frac{1+\alpha}{1-\alpha}$

It turns out that the stability is determined by the properties of the azimuthal field rather than the presence of a neutral point in the magnetic configuration. If $\alpha>1$, then one of the modes $(1,2)$ 
is unstable. On the contrary, one of the modes $(3,4)$ is unstable if $\alpha<1$. Note that the instability of the modes 3 or 4 is less efficient in the neighbourhood of the neutral point than in the rest volume (see below) because $\omega_{A}$ is small there and, hence, the growth time of instability is long.

Consider now the local stability of a region that is far off the neutral point. In this case, the term in $A$ proportional to $\delta$ can be neglected in Eq. (25). Then, we have

$\sigma^{4}+a_{2} \sigma^{2}+a_{0}=0$

where

$a_{2}=2 \omega_{A}^{2}+2 \mu \omega_{B}^{2}(1-\alpha), \quad a_{0}=\omega_{A}^{2}\left[\omega_{A}^{2}-2 \mu \omega_{B}^{2}(1+\alpha)\right]$.

Equation (29) has positive (unstable) roots if either $a_{2}<0$ or $a_{0}<0$. These two conditions are equivalent to

$\alpha>1+\left(k s_{1}\right)^{2} \frac{B_{z}^{2}}{\mu B_{\varphi}^{2}}$

and

$\alpha>-1+\left(k s_{1}\right)^{2} \frac{B_{z}^{2}}{2 \mu B_{\varphi}^{2}}$,

respectively. Obviously, if condition (31) is fulfilled, then condition (30) is also satisfied. Therefore, the true criterion of instability is given by Eq. (31). Depending on the parameters, the sufficient condition of instability (31) can differ substantially from the necessary condition (1) that is equivalent to $\alpha>-1$. The roots of Eq. (29) are

$\sigma^{2}=-\omega_{A}^{2}-\mu \omega_{B}^{2}(1-\alpha) \pm \sqrt{\mu^{2} \omega_{B}^{4}(1-\alpha)^{2}+4 \mu \omega_{A}^{2} \omega_{B}^{2}}$

In the case of a weak axial field $\left(\omega_{B}>\omega_{A}\right.$ or $\left.B_{\varphi}>\left(k s_{1}\right) B_{z}\right)$, the behaviour of instability is rather different for $\alpha>1$ and $\alpha<1$. If $\alpha>1$, the unstable root of Eq. (32) is equal to

$\sigma \approx \omega_{B} \sqrt{2 \mu(\alpha-1)}$

In this case, the instability grows on the timescale of the order of the time it takes an Alfvén wave in the field $B_{\varphi}$ to travel to the distance $s_{1}$. This regime recalls the instability of a purely toroidal field that occurs only at $\alpha>1$ and grows on the same timescale $\sim 1 / \omega_{B}$ (Braithwaite $\&$ Nordlund 2006). If $\alpha<1$, then the root corresponding to instability is given by

$\sigma \approx \sqrt{\frac{1+\alpha}{1-\alpha}} \omega_{A}$

For such profiles of the toroidal field, the instability essentially grows slower. Its growth time is of the order of an inverse Alfvén frequency associated with the axial magnetic field, $B_{z}$. This instability is less efficient in a relative neighbourhood of the neutral point where $\omega_{A}$ is small. Note that the value $\alpha \approx 1$ distinguishes between two regimes only in the limit of weak $B_{z}$. If $B_{z}$ is stronger, then the distinguishing value becomes higher.

Note that, in the case $\alpha=1$, Eq. (32) recovers the dispersion relation (19). If the axial magnetic field is sufficiently strong, axisymmetric perturbations are stable for any profile $B_{\varphi}(s)$.

\section{Numerical results}

In this section we discuss the stability of the magnetic configurations containing axial and azimuthal fields by numerically solving the eigenvalue problem. In calculations, we assume that the dependence of the azimuthal magnetic field on $s$ is given by

$B_{\varphi}=B_{\varphi 0}\left(\frac{s}{s_{1}}\right)^{\alpha}$

where $B_{\varphi 0}$ is the field strength at the inner boundary. To calculate the growth rate of the instability, it is convenient to introduce dimensionless quantities

$x=\frac{s}{s_{1}}, \quad q=k s_{1}, \Gamma=\frac{\sigma}{\omega_{B 0}}, \omega_{B 0}=\frac{B_{\varphi 0}}{s_{1} \sqrt{4 \pi \rho}}, \varepsilon=\frac{B_{z}}{B_{\varphi 0}}$.

Then, Eq. (11) transforms into

$$
\begin{aligned}
& \frac{\mathrm{d}}{\mathrm{d} x}\left(\frac{\mathrm{d} v_{1 s}}{\mathrm{~d} x}+\frac{v_{1 s}}{x}\right)-q^{2}\left\{1-x^{2(\alpha-1)}\left[\frac{4 q^{2} \varepsilon^{2}}{\left(\Gamma^{2}+q^{2} \varepsilon^{2}\right)^{2}}\right.\right. \\
& \left.\left.-\frac{2(1-\alpha)}{\Gamma^{2}+q^{2} \varepsilon^{2}}\right]\right\} v_{1 s}=-\frac{2 \delta}{x} \frac{q^{2} \varepsilon^{2}}{\Gamma^{2}+q^{2} \varepsilon^{2}}\left(\frac{\partial v_{1 s}}{\partial x}+\frac{v_{1 s}}{x}\right) .
\end{aligned}
$$

The parameter $\varepsilon$ can depend on $s$ in this equation since $B_{z}$ is generally a function of $s$ in our model. The inner and outer boundaries correspond to $x_{1}=1$ and $x_{2}=2$, respectively, where we have $v_{1 s}\left(x_{1}\right)=v_{1 s}\left(x_{2}\right)=0$. Equation (36), together with the given boundary conditions at the extrema, is a two-point boundary value problem that can be solved by using the "shooting" method (Press et al. 1992). In particular in order to solve Eq. (36), we used a fifth-order Runge-Kutta integrator embedded in a globally convergent Newton-Rawson iterator. As the spectrum is discrete, we first found the lowest eigenvalue for the analytical solution (19), and then we gradually changed $q$, $B_{z} / B_{\varphi}$ and $\alpha$ in order to explore the parameter space. In this way we checked that the eigenvalue was always the fundamental one, as the corresponding eigenfunction had no zero except the one at the boundaries. Higher radial wavenumbers corresponds to lower growth rates, as it is also apparent from Eqs. (20), (21).

The radial dependence of the eigenfunctions is shown in Fig. 1 for $\alpha=2$ and $B_{z} / B_{\varphi}=0.5(\delta=0)$. The curve corresponding to $q=1$ describes the unstable mode, but the other 3 curves represent stable modes. The eigenfunctions for $q<1$, representing unstable modes as well, almost coincide with the curve $q=1$ and are not shown in Fig. 1. Note that, for other values of the parameters, the unstable eigenfunctions can also have sharp maximum near the outer (if $\alpha>0$ ) or inner (if $\alpha<0$ ) boundaries. It seems that the maxima tend usually to be located near the boundary with the strongest field.

In Fig. 2, we plot $\Gamma=\sigma / \omega_{B 0}$ as a function of $q=k s_{1}$ for a weak constant axial field. The addition of even very weak $B_{z}$ changes the stability properties substantially. A purely toroidal field should be unstable if $\alpha>1$. Our calculations show, however, that even the profile of the toroidal field with $\alpha=0$ is unstable in this case despite a very low energy of the axial field compared to that of the toroidal field $(\sim 1 \%)$. It is also worth noticing an important difference between the necessary condition of instability (1) and the sufficient condition. In accordance with inequality (1), the necessary condition requires $\alpha>\alpha_{\mathrm{c}}=-1$, but our calculations show that the instability occurs at essentially larger $\alpha_{\mathrm{c}} \sim-0.1$. The growth rate as a function of $q$ always has a smoothed maximum at $q \sim 5-20$ depending on $\alpha$. The value of $q$ corresponding to the maximum increases with increasing $\alpha$. The figure clearly illustrates the difference between the two regimes 


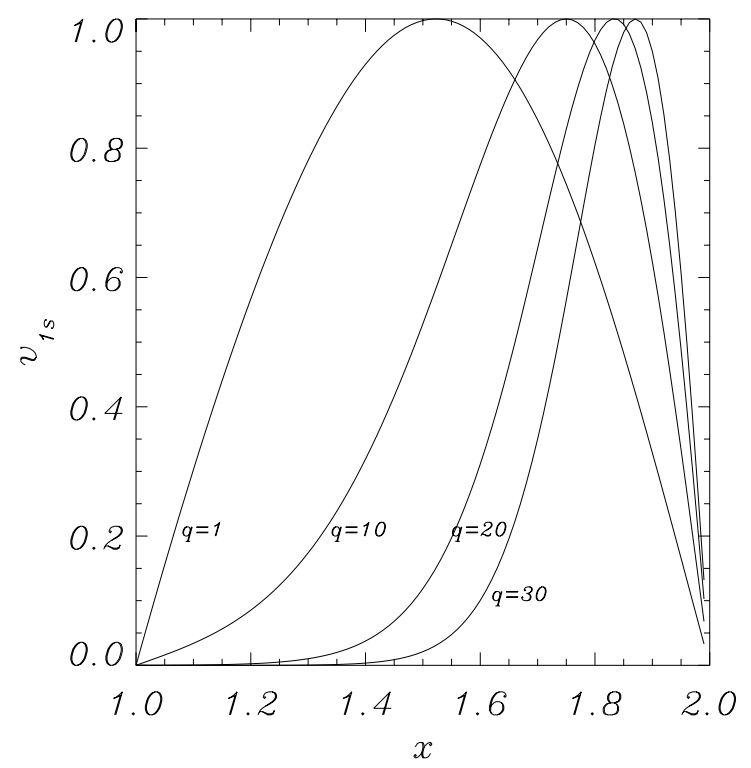

Fig. 1. The dependence of the fundamental eigenfunctions on $s$ for $\alpha=$ $2, B_{z} / B_{\varphi 0}=0.5$, and several values of $q$.

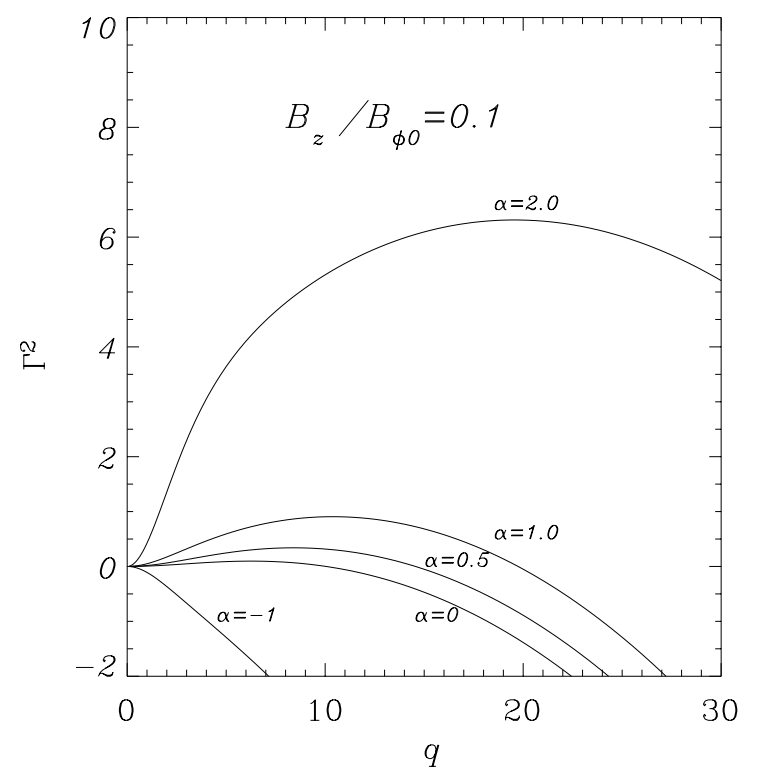

Fig. 2. The dependence of $\Gamma^{2}$ on $q$ for $B_{z} / B_{\varphi 0}=0.1$ and several values of $\alpha$.

of the instability discussed in Sect. 4. If $\alpha<1$, the instability is rather weak and grows on the Alfvén timescale characterised by the axial field. In contrast, if $\alpha>1$, the instability is much more efficient, and the growth time is close to the Alfvén timescale for the toroidal field.

The dependence of $\Gamma^{2}$ on $q$ for a stronger axial field is shown in Fig. 3. An increase of the axial field makes the magnetic configuration more stable. For example, in the case $\alpha=2$, the maximum growth rate of instability is $\approx 2.5 \omega_{B 0}$ if $\varepsilon=0.1$, but it only becomes $\approx 1.7 \omega_{B 0}$ if $\varepsilon=0.5$. As mentioned, the critical value of $\alpha$ that determines the onset of instability is $\sim-0.1$ in the case $\varepsilon=0.1$. If $\varepsilon=0.5$, then instability can occur only if the toroidal field increases enough rapidly with $s$, and the critical value $\alpha_{\mathrm{c}}$ is close to 1 . In a stronger axial field, the transition between two regimes of instability (see Eqs. (30) and (31)) occurs at a higher value of $\alpha$ as was predicted in Sect. 3.2.

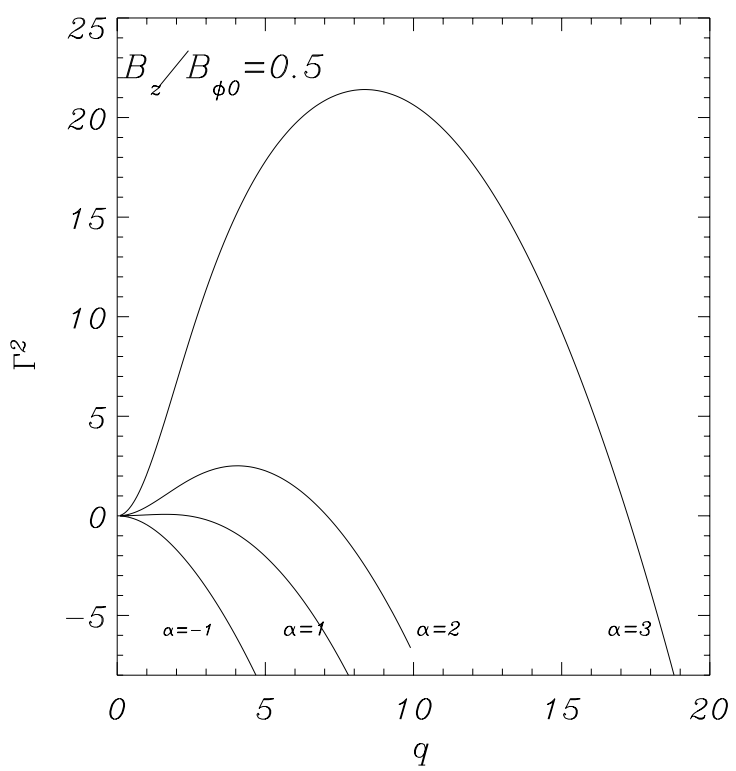

Fig. 3. The same as in Fig. 2 but for $B_{z} / B_{\varphi 0}=0.5$.

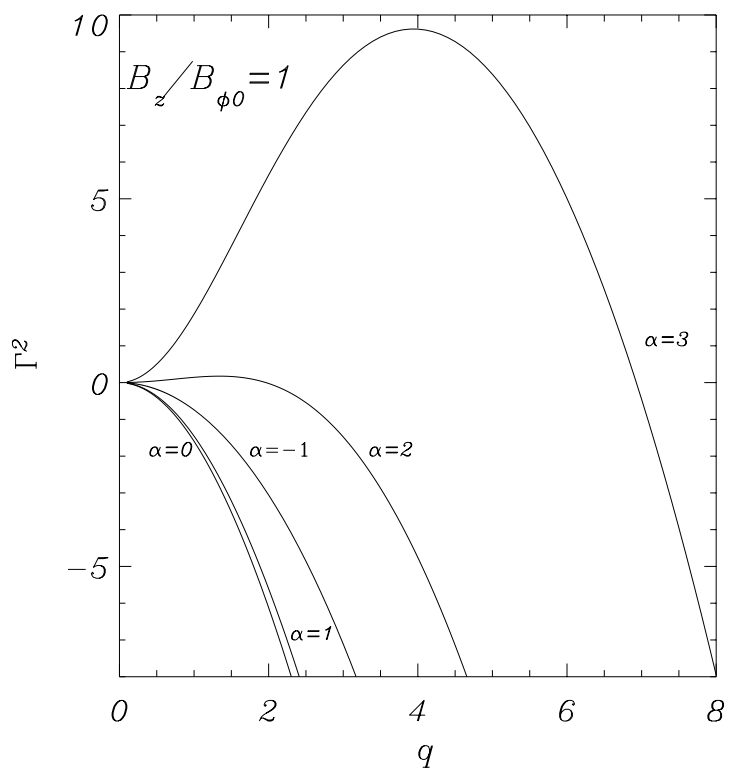

Fig. 4. The same as in Fig. 2 but for $B_{z} / B_{\varphi 0}=1$.

In Fig. 4, the normalized growth rate is shown for $B_{z} / B_{\varphi}=1$. Such a strong axial field stabilises the magnetic configuration drastically. The instability occurs only if the toroidal field increases very rapidly with $s(\alpha \geq 2)$. A qualitative behaviour of unstable eigenvalues is the same as in Figs. 2 and 3, but the maximum growth rate corresponds to lower values of $q$.

In Fig. 5, we plot the dependence of $\Gamma^{2}$ on $\varepsilon=B_{z} / B_{\varphi 0}$ for $q=1$. The profiles of the toroidal field with $\alpha=0.5$ and 1 are stable if $B_{z}=0$. Therefore, these curves start with $\Gamma^{2}=0$. In contrast, the toroidal field profile with $\alpha=2$ is unstable even if the axial field is vanishing and, therefore, $\Gamma^{2}(\varepsilon=0) \neq 0$. All the curves shown in Fig. 5 exhibit a qualitatively similar behaviour: the growth rate reaches a rather flat maximum at $B_{z} / B_{\varphi 0} \approx 0.3-0.5$ and then decreases fast enough. The quantity $\Gamma^{2}$ becomes negative and, hence, the magnetic configuration is stable if $B_{z}$ is sufficiently strong. The higher is the value of $\alpha$, the stronger the axial field required for stabilization. 


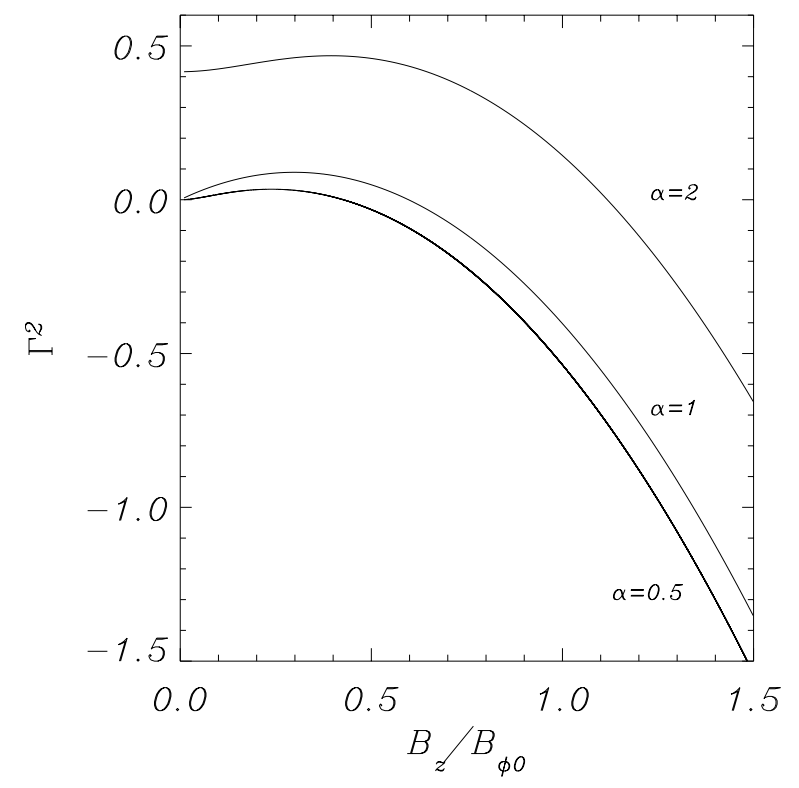

Fig. 5. The dependence of $\Gamma^{2}$ on $B_{z} / B_{\varphi 0}=1$ for $q=1$ and different values of $\alpha$.

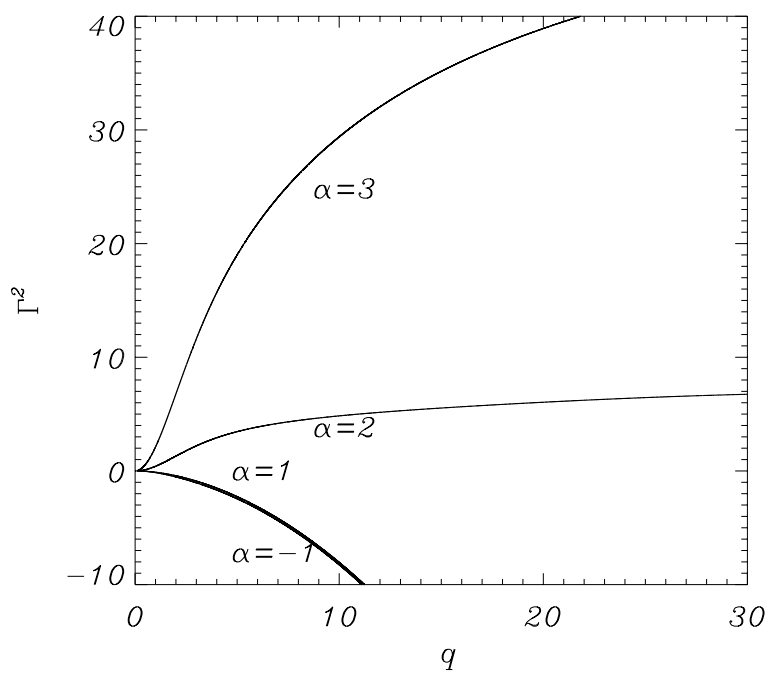

Fig. 6. The same as in Fig. 2 but for $B_{z}$ given by Eq. (38) with $x_{0}=1.75$ and $B_{z 0} / B_{\varphi 0}=0.6$.

In Fig. 6, we plot the dependence of $\Gamma^{2}$ on $q$ for the axial magnetic field that depends on $s$. We choose this dependence in the form

$B_{z}(s)=B_{z 0}\left(1-\frac{s}{s_{0}}\right)=B_{z 0}\left(1-\frac{x}{x_{0}}\right)$

where $x_{0}=s_{0} / s_{1}, s_{0}$ is a radius at which $B_{z}$ changes sign, $s_{2}>$ $s_{0}>s_{1}$. We have for such $B_{z}$

$\delta=\frac{x}{x-x_{0}}, \quad \varepsilon=\varepsilon_{0} \frac{x_{0}-x}{x_{0}}$,

where $\varepsilon_{0}=B_{z 0} / B_{\varphi 0}$. Calculating models in Fig. 6, we suppose $x_{0}=1.75$ and $\varepsilon_{0}=0.6$. The ratio $B_{z} / B_{\varphi 0}$ averaged over $s$ is equal to 0.1 in this case, thus the results can be compared with those in Fig. 2 where $B_{z}=$ const. and $B_{z} / B_{\varphi 0}=0.1$. Although the axial field has the neutral line at $s_{0}=1.75$, the stability properties are not very different from the similar configuration with no neutral

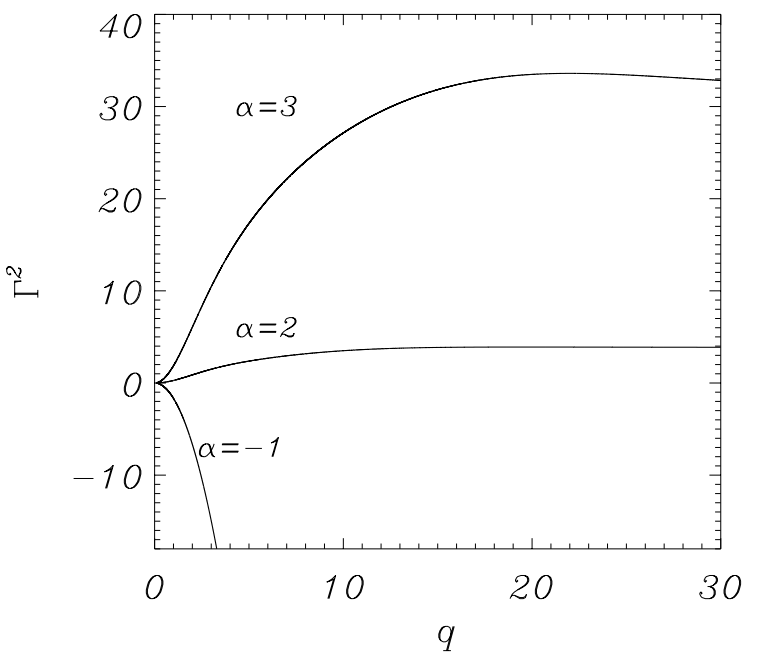

Fig. 7. The same as in Fig. 6 but for $B_{z 0} / B_{\varphi 0}=3$.

line (Fig. 2). In Fig. 6, the critical value of $\alpha$ that distinguishes between stable and unstable configurations is close to 0 . If the axial field has no neutral line (Fig. 2), then the critical value is fairly close to this value ( - 0.1). The maximum growth rate is of the same order of magnitude for the same $\alpha$. The only difference is that configurations with the neutral line (Fig. 6) are unstable for a wider range of $q$.

In Fig. 7, we plot $\Gamma^{2}$ versus $q$ for the case of a stronger axial field $\left(B_{z 0} / B_{\varphi 0}=3\right)$ with the same neutral line at $s_{0}=1.75$. The qualititative behaviour is similar to what is depicted in Fig. 6, although the growth rates are lower because of the higher value of $B_{z 0} / B_{\varphi 0}$. The averaged ratio $B_{z} / B_{\varphi 0}$ is 0.5 in this case, and the results can be compared to Fig. 3 where $B_{z} / B_{\varphi 0}=0$. It appears that the presence of a neutral line does not change the stability properties substantially. For instance, the critical value of $\alpha$ is $\sim 1$ for both configurations. The maximum growth rate at a given $\alpha$ is of the same order of magnitude as well. However, the rage of unstable $q$ is wider for configurations with the neutral line.

\section{Discussion}

We have considered the hydromagnetic stability of cylindrical configurations containing the toroidal and axial magnetic fields. Dissipative effects were neglected in our study. We treated a linear stability assuming that the behaviour of small perturbations is governed by equations of incompressible hydrodynamics. This approximation is justified if the magnetic field is subthermal and the Alfvén velocity is low compared to the sound speed. The stability of the magnetic configurations is a key issue for understanding the properties of various astrophysical bodies, such as peculiar A and B stars, magnetic white dwarfs, neutron stars, etc. Even though various dynamo models predict that the toroidal field should be typically stronger than the poloidal one, the effect of a poloidal field on the stability usually cannot be neglected.

To demonstrate this, we treated the simplest model of a highly conducting fluid between two cylindrical surfaces. We assumed that the toroidal and axial fields depends on the cylindrical radius alone. In a short-wavelength approximation, we derived the growth rate and a sufficient criterion of instability analytically (Eqs. (30), (31)). For large-scale perturbations, the condition of instability and its growth rate were calculated 
numerically. The analytical and numerical results are in good qualitative agreement. The obtained conditions of instability differ substantially from what is predicted by the necessary condition (1). For instance, according to Eq. (1), if the instability occurs in the magnetic configuration, then the toroidal field profile satisfies the condition $\alpha>-1$. In fact, the instability occurs only if the toroidal field decreases with $s$ much slower (or even increases): the critical value of $\alpha$ is $\approx-0.1$ if $B_{z} / B_{\varphi 0}=0.1$ and $\approx 1$ if $B_{z} / B_{\varphi 0}=0.5$. If $B_{z}$ depends on $s$, then the critical values of $\alpha$ should be even higher.

Depending on the profile of the toroidal field and the strength of the axial field, the instability can arise in two essentially different regimes. In the case of a weak axial field, $B_{\varphi 0} \gg B_{z}$, the value of $\alpha$ that distinguishes between the regimes is $\approx 1$. If $\alpha>1$, then the instability grows on the Alfvén timescale determined by the toroidal field and is rather fast. If $\alpha<1$, then the instability is much slower and grows on the timescale determined by the axial field. The transition between these two regimes occurs at larger $\alpha$ if the axial field increases. The efficiency of the considered instability turns out to be rather low if $\alpha<1$ and $B_{z}$ is weak.

It is worth noticing the very particular properties of instability in the case $\alpha \approx 1$. In such a configuration, the particular type of MHD waves is given by the dispersion Eq. (19). The growth rate of these waves (or the frequency, if the waves are stable) is proportional to the product of $B_{z}$ and $B_{\varphi}$. These waves cannot exist in purely toroidal or purely poloidal fields. The instability of the configuration with $\alpha \approx 1$ is caused by the generation of this particular type of wave. These waves can probably determine the instability of magnetic configurations near the axis of symmetry where $B_{\varphi} \rightarrow 0$.

A sufficiently strong axial field always suppresses the instability. For more or less plausible values of $\alpha \leq 1$, the strength of the axial field stabilising the configuration is $\sim 0.1-1 B_{\varphi}$. A much stronger field is required, however, to stabilise the configuration with larger $\alpha$.
Acknowledgements. This research project was supported by a Marie Curie Transfer of Knowledge Fellowship of the European Community's Sixth Programme under contract number MTKD-CT-002995. V.U. thanks also INAFOssevatorio Astrofisico di Catania for hospitality.

\section{References}

Acheson, D. 1973, J. Fluid Mech., 61, 609

Acheson, D. 1978, Phil. Trans. R. Soc. London, 289A, 459

Barnes, G., Charbonneau, P., \& MacGregor, K. 1999, ApJ, 511, 466

Bonanno, A., \& Urpin, V. 2006, Phys. Rev. E, 73, 066301

Borra, E., Landstreet, J., \& Mestel, L. 1982, ARA\&A, 20, 191

Braithwaite, J. 2006, A\&A, 453, 687

Braithwaite, J., \& Spruit, H. 2004, Nature, 431, 819

Braithwaite, J., \& Nordlund, A. 2006, A\&A, 450, 1077

Braithwaite, J., \& Spruit, H. 2006, A\&A, 450, 1097

Chandrasekhar, S. 1960, Proc. Natl. Acad. Sci., 46, 253

Chanmugam, G. 1979, MNRAS, 187, 769

Dubrulle, B., \& Knobloch, E. 1993, A\&A, 274, 667

Gilman, P. A. 1970, ApJ, 162, 1019

Howard, L., \& Gupta, A. 1962, J. Fluid Mech., 14, 463

Kitchatinov, L. L., \& Rüdiger, G. 2007 [arXiv:astro-ph/0701847]

Knobloch, E. 1992, MNRAS, 255, 25

Markey, P., \& Tayler, R. 1973, MNRAS, 163, 77

Markey, P., \& Tayler, R. 1974, MNRAS, 168, 505

Mestel, L. 1999, Stellar Magnetism (Oxford: Clarendon)

Morse, P., \& Feshbach, H. 1953, Methods of Theoretical Physics (New York: McGraw-Hill)

Olver, F. W. J. 1970, in Handbook of Mathematical Functions, ed. M. Abramovitz, \& I. Stegun (New York: Dover Publications)

Papaloizou, J., \& Terquem, C. 1997, MNRAS, 287, 771

Parker, E. N. 1955, ApJ, 121, 491

Prendergast, K. 1956, ApJ, 123, 498

Press, W. H., Teukolsky, S. A., Vetterling, W. T., \& Flannery, B. P. 1992, Numerical Recipies in FORTRAN, The art of scientific computing (Cambridge: UP)

Spruit, H. 1999, A\&A, 349, 189

Terquem, C., \& Papaloizou, J. 1996, MNRAS, 279, 767

Tayler, R. J. 1973, MNRAS, 161, 365

Tayler, R. J. 1980, MNRAS, 191, 151

Van Assche, W., Tayler, R. J., \& Goosens, M. 1982, A\&A, 1966

Velikhov, E. P. 1959, Sov. Phys. JETP, 9,995

Wright, G. A. E. 1973, MNRAS, 162, 339 